\title{
A Perspective in Antenatal Care: A Short Emphasis on Hydration, Essential Fatty Acids and Micronutrients
}

\author{
Mohammed Ayed Huneif ${ }^{1}$ and Ramadhan Oruch ${ }^{2 *}$ \\ ${ }^{1}$ Department of Pediatrics, School of Medicine, Najran University, Najran, Kingdom of \\ Saudi Arabia \\ ${ }^{2}$ Department of Biochemistry and Molecular Biology, School of Medicine, Najran \\ University, Najran, Kingdom of Saudi Arabia \\ *Corresponding Author: Ramadhan Oruch, Department of Biochemistry and \\ Molecular Biology, School of Medicine, Najran University, Najran, Kingdom of Saudi \\ Arabia.
}

Received: March 19, 2021

Published: May 11, 2021

C All rights are reserved by Mohammed Ayed Huneif and Ramadhan Oruch.

\begin{abstract}
Antenatal care of women is a vital issue with regards to health promotion for forthcoming infants. This type of medical care should start before the onset of a pregnancy. Antenatal care facilitates early and continuous assessment of the potential health risks during pregnancy both for pregnant women and their fetuses. To achieve this, especially in primigravida women, a complete medical history should be recorded at the first prenatal visit. Control of chronic diseases such as diabetes, hypertension, and psychiatric ailments must be carried out. Healthy nutrition is important to re-enforce the immune system of pregnant women and thus aid their forthcoming infants to fight infection. The aim of this work was to pinpoint the significance of healthy nutrition to antenatal caregivers in different maternity stations, junior doctors at medical centers, medical and nursing students and also pregnant women per se.
\end{abstract}

Keywords: Pregnancy; Antenatal Care; Lipids; PUFAs; Micronutrients; Fat Soluble Vitamins; Reactive Oxygen Species; Antioxidants; Neural Tube Defects

\section{Introduction}

Pregnancy, which is also called gestation, is a physiological event most women undergo once or more, in one stage or another, of their reproductive lives. This physiological event occurs normally in the uterus after coitus or assisted reproductive technology (induced pregnancy). A pregnancy may end in a live birth, abortion, or miscarriage (spontaneous abortion). If implantation of a fertilized ovum occurs outside the uterus then this is termed an ectopic pregnancy. This abnormal pregnancy usually ends with obstetrical complications that need surgical (gynecological) intervention.

Child birth typically occurs around 40 weeks from the start of the last menstrual period, with some variations in certain cases.
Early signs and symptoms of pregnancy briefly include: missed periods, tender breasts, nausea and emesis, feeling of hunger, frequent micturition. In late pregnancy, pelvic pain may also appear as a problem and it is then recommended to consult an obstetrician to seek advice. Pregnancy test is carried out to positively confirm a pregnancy, especially in the case of primigravida.

To assure a safe pregnancy, both for the sake of the pregnant woman and her coming neonate, correct prenatal care is necessary.

Antenatal care: Is indeed a preventive-type of health care, designated to provide obstetric check-up. This is usually carried out by midwives or physicians (first visit), to prevent or treat any potential health problems that might arise during the course of the 
pregnancy. The aim of these preventive measures is to promote a healthy lifestyle for the pregnant woman and her forthcoming child. These checkups can occur at different intervals according to the welfare and health facilities of different countries.

The importance of antenatal care lies in the fact that the pregnant individual will receive beneficial medical information relating to physiological and biological changes occurring in their bodies and also details on prenatal nutrition including micronutrients and other vital (essential) food components that cannot be biosynthesized in the human body. Despite controversy as regards vaccination programs for pregnant women, it is accepted as one of the corner preventive procedures to protect the developing fetus from a dozen or so complications such as congenital malformation. Use of unnecessary drugs should be avoided, especially in the $1^{\text {st }}$ trimester of pregnancy since certain drugs can predispose to congenital malformations and other neurological diseases. Among these drugs valproate [1,2] and benzodiazepines [3] are well known.

Psychological evaluation and advice might be necessary to inform primigravida women about the burdens of pregnancy on her body, and also on her relations to her spouse and the rest of her family members (in the case of a multiparous women). Psychological disturbances are usually attributed to hormonal changes that a pregnant woman experiences during the gestational period. Obstetric care-personal need to be aware that some of these psychological problems might continue even after birth and cause what is known as puerperal blues. In most cases professional psychiatric intervention is needed.

A report from the World Health Organization (WHO) in 2015 declared that around 830 females died daily because of gestational complications and child birth, the majority of which were in the $3^{\text {rd }}$ world [4]. The recommendations of WHO, state that pregnant women should receive at least four antenatal visits to rule out problems associated with pregnancy. These visits should also include ultrasonic examination to diagnose as early as possible any potential complications that require treatment. The visits should also include the necessary immunization programme. What concerns us here is the nutritional status during pregnancy and its reciprocal impact on their coming babies.

\section{Discussion}

Hydration: There is great individual variation in the volume of liquid we imbibe and this is also true among pregnant women. However, the most significant issue here is that these women have to stay well hydrated. In general it is difficult to set a rule concerning how much fluid a pregnant woman should drink. One should bear in mind that the amount increases as the pregnancy advances towards end-term. Why is water so important? There are several reasons: It is needed to produce amniotic fluid that surrounds the fetus, helps to increase blood volume and is important in preparing the mammary glands to produce milk for the coming baby. Hyperemesis gravidarum (morning sickness) per se, where frequent vomiting occurs in certain pregnancies [5], is another reason why these women need to keep themselves well hydrated. The most significant parameter that warns pregnant women about their fluid requirement is thirst, so they should be advised to respond to this sign by increased fluid intake, the best of which is plain pure water, unless the situation says otherwise. All non-alcoholic drinks contribute to fluid intake, including milk, fruit juice, smoothies and hot drinks (a mug of tea $75 \mathrm{mg}$ of caffeine and/or a mug of instant coffee containing $100 \mathrm{mg}$ daily is acceptable but not more). To add sugar or not to these beverages is dependent on the situation, i.e. whether diabetes or gestational diabetes is a health issue. Another parameter that can be monitored with ease is urine color. Normal urine has a pale straw color, while dark colored urine indicates dehydration and this is thus a sign of a requirement for fluid intake. The message here is that pregnant women should be always well hydrated during the course of a normal pregnancy.

Lipids, fat soluble vitamins: Dietary lipids generally include the fatty acids that are naturally incorporated into monoglycerides, diglycerides, triglycerides, and phospholipids. It also included fat soluble vitamins (A, D, E, and $\mathrm{K}$ ). The most important functions of these biomolecules for human beings (pregnant women in this context) are: 1) energy storage, 2) structural composition of cell membranes, which is important for cytogenesis and cell proliferation in the fetus, 3 ) and storage of fat soluble vitamins within the adipose tissues in between triglyceride molecules. For a lipid to be utilized as a fuel material to produce the biological energy currency adenosine triphosphate (ATP), it needs to be degraded to its fatty acid components. These hydrolytic reactions occur via the process of lipolysis which is catalyzed by different types of lipases. The advantage of fatty acids (FAs) over sugars (glucose) as fuel molecules is that a given amount of FA produces more energy than the same amount of glucose, though glucose could be utilized both aerobically and anaerobically. Glucose can be oxidized both in

Citation: Mohammed Ayed Huneif and Ramadhan Oruch. "A Perspective in Antenatal Care: A Short Emphasis on Hydration, Essential Fatty Acids and Micronutrients". Acta Scientific Paediatrics 4.6 (2021): 18-23. 
cytoplasm (anaerobic glycolytic pathway) and aerobically in mitochondria, while FAs are beta oxidized in mitochondria only under aerobic conditions. Oxidation of FAs is accompanied by a release of free radicals (reactive oxygen species) as a byproduct. These unstable radicals (molecular particles) can affect cellular macromolecules such as proteins, DNA, and even the phospholipids of biomembranes. Integrity of biomembranes is vital to have healthy and sound cells, including those vulnerable cells of the fetus (inner cell mass in the first trimester), as well as in the stage of neural tube development and the stage of organogenesis later on. Vitamin E (tocopherol) is the one devoted to protect cell membranes as this micronutrient is fat soluble and associates with phospholipid leaflets of neuronal biomembranes [6]. Turnover of phospholipids i.e. biosynthesis and degradation, is under strict and tightly controlled balance. The enzymes that degrade membranal phospholipids are known as phospholipases and these are indeed many in number. One important enzyme is phospholipase A2 or $\left(\mathrm{PLA}_{2}\right)$, especially the subtype cytosolic phospholipase $\mathrm{A} 2\left(\mathrm{CPLA}_{2}\right)$ is worthy of mention. The importance of this protein is that it catalyzes the cleavage of fatty acid tail $S N-2$ from the phosphoglycerol molecules, leading to formation of lysophosphatidic acid and a free fatty acid [7]. The detached free fatty acid (after cleavage) is usually of unsaturated type, mostly of omega-3, omega-6 types or omega-9 polyunsaturated fatty acids (PUFAs). These PUFAs are vital to maintain membrane fluidity so that receptors can be kept embedded in these biomembranes in correct alignment in order to keep the signal transduction processes intact and ongoing normally. This is important and a fundamental requirement for normal neuronal function and also other somatic cells of both pregnant women and their fetuses (coming babies). Once these PUFAs are released, they rapidly catalyze the biosynthesis of other vital metabolites, for example the PUFA arachidonic acid, by the downstream modification by proteins such as cyclooxygenases (lipoxygenases) into eicosanoids. These primary metabolites which include prostaglandins and leukotrienes, have anti-inflammatory and inflammatory functions within the human body respectively. For a pregnant woman to keep her fetus, and indeed her own body in optimum health, she should have a rich pool of PUFAs in her body, since these molecules have very high turnover rates including both apoptotic and cytogenetic categories. When a PUFA is cleaved, it should normally be substituted with another PUFA (if available), otherwise $S N-2$ position of membranal phosphoglycerides will be occupied by a saturated one (fatty acid). This process will decrease membrane fluidity and affect many metabolic, biosynthetic and signal transduction processes attributed to receptor misalignment in these biomembranes (membrane pacemaker theory) [8]. The most significant and wellknown function of PUFAs, according to the membrane pace maker theory, is that they decrease the risk of metabolic syndrome [9]. The spectrum of this syndrome includes obesity, hypercholesterolemia, and hypertension and type II diabetes. All these parameters affect the fetus and cause post-partum complications in pregnancy. These complications include puerperal blues (depression and or psychosis), and many metabolic diseases such as diabetes may also affect these women in any future pregnancies. PUFAs prevent a majority, if not all of these complications that may affect women postpartum and secure the delivery of sound babies both neurologically and somatically (Apgar score 7 - 10). Type II diabetes mellitus, and gestational diabetes as a secondary factor, can result in large babies (macrosomia) and complications during delivery [10]. PUFAs have also a vital role to play in treatment/prevention of many cases of the ADHD spectrum of diseases in children [11,12]. Taken together these polyunsaturated fatty acids were previously called vitamin F. The term "vitamin" was used because omega-3 (such as: $\alpha$-linolenic acid and docosahexaenoic acid) and omega-6 (such as: linoleic acid and arachidonic acid) are essential micronutrients in that they cannot be biosynthesized in the human body, in other words, they must be provided by the diet (usually sea food). To our knowledge the provision of pregnant women with cod-liver oil which includes these essential nutrients (and the fat soluble vitamins $A$ and $D$ ) is a routine practice in the Scandinavian countries and in the Western world in general. An adequate supply of vitamin A (and its vitamers) is especially important for pregnant and breast feeding women, both for normal fetal development and breast feeding of coming neonates. Antenatal care-providers need to bear in mind that vitamin A deficiency cannot be compensated by postnatal dietary supplementation $[13,14]$. Vitamin A has important biological roles in the human body including its function as a fat soluble antioxidant [15]. On the other hand we must remember that an excess of this fat-soluble metabolite can cause hypervitaminosis A (vitamin A toxicity). Vitamin A toxicity can cause birth defects, thus supplementation should not exceed the recommended daily value, which is equivalent to 750 - $1300 \mu \mathrm{g}$ retinol $[16,17]$.

Concerning vitamin D deficiency, this is common in pregnant women because they often do not get the recommended amount of this in their daily diet $[18,19]$. Obesity is a potential factor that de- 
creases serum levels of vitamin D. A meta-analysis from 2016 pinpointed that circulating vitamin D status was improved by weight loss. Similarly, serum vitamin D levels in women may show an inverse relationship during pregnancy [20]. Vitamin D deficiency was found to be associated with gestational diabetes, preeclampsia and "small for date" infants [21-23]. Although vitamin D supplementation during pregnancy raises serum level of vitamin $\mathrm{D}$, the potential benefit of this on the mother or on their babies is unclear [24]. Women should always be prepared for stressful times in their lives, especially during the reproductive period, such as pregnancy. When they have plans to become pregnant, they should be well prepared as early as possible for this event, both somatically and psychologically. It is an accepted scientific fact among obstetricians that women who take an adequate amount of this fat soluble vitamin during gestation may experience a lower risk of preeclampsia and secure an effective immune system [18]. The recommended daily intake of vitamin $\mathrm{D}$ in pregnancy and lactation varies in different countries, but generally ranges between 15 - $100 \mu \mathrm{g}$, where $100 \mu \mathrm{g}$ is the generally recognized upper tolerable intake value.

The routine antenatal care programme in developing countries should also include supplementations of iron, folic acid and vita$\min \mathrm{B}_{12}$.

Concerning folate (folic acid; vitamin $\mathrm{B}_{9}$ ), deficiency of this essential water soluble vitamin has been implicated in in neural tube defects (NTDs). Before implementation of food fortification policy of this essential metabolite in many countries, the estimated number of cases worldwide was about 300,000 [25]. It is important that pregnant women should take this vitamin early in pregnancy because NTDs can namely occur during the $1^{\text {st }}$ month. Abundant intake of this supplement is therefore necessary before and during pregnancy, especially for women in developing countries [26]. According to the US National Institutes of Health the recommended daily allowance of this metabolite for pregnant women is $600 \mu \mathrm{g}$ [27].

Another water soluble essential metabolite, namely a vitamer within the vitamin B-complex family, is riboflavin (vitamin $\mathrm{B}_{2}$ ). Deficiency of this micronutrient can result in birth defects manifested as congenital malformation such as congenital heart defects and limb deformities [28,29]. The recommended daily allowance during pregnancy lies between 1.4 and $1.6 \mathrm{mg}$ [27]. Minerals are important in antenatal care, including selenium. This element has an antioxidant function and can be provided via a diet that includes tuna fish, a very rich dietary source of selenium. Vitamin C (L-ascorbic acid), which exists abundantly in fresh citrus fruits, tomatoes and fresh vegetables, is also important in three respects, firstly: it is the universal antioxidant that reduces other antioxidants in the human body such as the fat soluble vitamins $\mathrm{E}$ and $\mathrm{A}$, secondly: this is a water soluble molecule that functions within the body fluids and cytosol of different cells in human tissues, thirdly: it enhances absorption of iron from the gastrointestinal tract and helps in wound healing. All these are important processes that need to be active in pregnant women.

\section{Conclusion}

As has been highlighted here, antenatal care is a pivotal preventive measure to protect neonates from different diseases postnatally and to decrease the possibilities of congenital malformations that might arise because of ignorance or shortage of antenatal care. It also functions to protect women in the postnatal phase, especially during the puerperal period, from different psychologic and somatic diseases that often affect this group of women. Lactation is also another burden on the mother and this is why their nutritional status should always be at an optimum in order to assure that they can perform this task properly. To have well-trained maternity care-givers is an important issue to assist mothers in the period of puerperium. Women in this period are liable to develop malnutrition especially of the undernutrition type. This is usually attributed to socioeconomic factors such poverty as is the case in certain $3^{\text {rd }}$ world societies or ignorance in more developed and prosperous societies. Alcohol and Illegal drug use such as psychoactive agents or hypnotics by women in certain societies are additional factors related to an ignorance of healthy life-style and thus nutritional health. Mental ailments such as major depression, bipolar affective disorders or psychosis, are other factors causing decreased self-esteem and ignorance of healthy and proper nutrition. In many cases proper nutrition can alleviate symptoms and signs of these morbidities as has been stated elsewhere in this mini-review. Proper antenatal care should also be outlined before advising ladies to get pregnant. Healthy nutrition that includes a diet rich in vitamins and minerals and containing antioxidants to scavenge free radicals from tissues and cells of pregnant women, and thus also their fetuses, is an important preventive measure that can provide protection against many diseases. This is because free radicals are 
a corner-stone predisposing factor to many diseases both somatic and mental. Healthy nutrition must include sufficient amounts of protein, different micronutrients (vitamins, minerals) and essential fatty acids as these are necessary components of a healthy daily diet recommended for pregnant women. Together with adequate hydration these dietary nutrients work hand in hand to promote a normal pregnancy and secure the delivery of healthy neonates.

\section{Acknowledgements}

Authors like to thank Prof. Dr. Med. Hamdan Sirag from the department of gynecology and obstetrics at the school of medicine for the preliminary checking and reviewing the manuscript.

\section{Conflicts of Interest}

None.

\section{Bibliography}

1. Mawhinney E., et al. "Valproate and the risk for congenital malformations: Is formulation and dosage regime important?" Seizure 21.3 (2012): 215-218.

2. Robinson CJ., et al. "Association of maternal vitamin D and placenta growth factor with the diagnosis of early onset severe preeclampsia". American Journal of Perinatology 30.3 (2013): 167-172.

3. Uzun S., et al. "Side effects of treatment with benzodiazepines". Psychiatria Danubina 22.1 (2010): 90-93.

4. Malik YK., et al. "Olanzapine-induced leucopaenia and thrombocytopaenia in an elderly patient: a case report and review of the evidence". General Psychiatry 31.2 (2018): e000013.

5. Verberg MF., et al. "Hyperemesis gravidarum, a literature review”. Human Reproduction Update 11.5 (2005): 527-539.

6. Oruch R., et al. “Global Journal Of Biochemistry 28 Global Journal of Biochemistry | Volume 2 | Issue 1 | March 2011 The fat soluble antioxidant vitamin E: Its metabolism, and biological and physiological significance". Global Journal of Biochemistry 2 (2011): 28-48.

7. Oruch R., et al. "Effects of psychotropic drugs on the thrombininduced liberation of arachidonate in human platelets". Saudi Medical Journal 29.10 (2008): 1397-13407.

8. Hulbert AJ. "The links between membrane composition, metabolic rate and lifespan". Comparative Biochemistry and Physiology Part A: Molecular and Integrative Physiology 150.2 (2008): 196-203.
9. Hulbert AJ. "Metabolism and longevity: is there a role for membrane fatty acids?" Integrative and Comparative Biology 50.5 (2010): 808-817.

10. Kuc S., et al. "Prediction of macrosomia at birth in type-1 and 2 diabetic pregnancies with biomarkers of early placentation". An International Journal of Obstetrics and Gynaecology 118.6 (2011): 748-754.

11. Agostoni C., et al. "The Role of Omega-3 Fatty Acids in Developmental Psychopathology: A Systematic Review on Early Psychosis, Autism, and ADHD". International Journal of Molecular Sciences 18.12 (2017): 2608.

12. Ciappolino V., et al. "The role of $n-3$ polyunsaturated fatty acids (n-3PUFAs) in affective disorders". Journal of Affective Disorders 224 (2017): 32-47.

13. Schulz C., et al. "Vitamin A and beta-carotene supply of women with gemini or short birth intervals: a pilot study". European Journal of Nutrition 46.1 (2007): 12-20.

14. Strobel M., et al. "The importance of beta-carotene as a source of vitamin A with special regard to pregnant and breastfeeding women". European Journal of Nutrition 46.1 (2007): I1-20.

15. Oruch R and Pryme I. "The biological significance of vitamin A in humans: A review of nutritional aspects and clinical considerations". Science Jet 1 (2012): 1-13.

16. Dietary Reference Intakes for Vitamin A, Vitamin K, Arsenic, Boron, Chromium, Copper, Iodine, Iron, Manganese, Molybdenum, Nickel, Silicon, Vanadium, and Zinc, Food and Nutrition Board of the Institute of Medicine, pages (2001): 82-161.

17. Deltour L., et al. "Ethanol inhibition of retinoic acid synthesis as a potential mechanism for fetal alcohol syndrome". The FASEB Journal 10.9 (1996): 1050-1057.

18. Wagner CL., et al. "Vitamin D and its role during pregnancy in attaining optimal health of mother and fetus". Nutrients 4.3 (2012): 208-230.

19. Wagner CL., et al. "The role of vitamin D in pregnancy and lactation: emerging concepts". Womens Health 8.3 (2012): 323340.

20. Mallard SR., et al. "Vitamin D status and weight loss: a systematic review and meta-analysis of randomized and nonrandomized controlled weight-loss trials". The American Journal of Clinical Nutrition 104.4 (2016): 1151-1159. 
21. Aghajafari F., et al. "Association between maternal serum 25-hydroxyvitamin D level and pregnancy and neonatal outcomes: systematic review and meta-analysis of observational studies". British Medical Journal 346 (2013): f1169.

22. Palacios C., et al. "Vitamin D supplementation for women during pregnancy". The Cochrane Database of Systematic Reviews 7 (2019): CD008873.

23. Palacios C., et al. "Regimens of vitamin D supplementation for women during pregnancy". The Cochrane Database of Systematic Reviews 10 (2019): CD013446.

24. Roth DE., et al. "Vitamin D supplementation during pregnancy: state of the evidence from a systematic review of randomised trials". British Medical Journal 359 (2017): j5237.

25. Berry RJ., et al. "Fortification of flour with folic acid". The Food and Nutrition Bulletin 31.1 (2010): S22-35.

26. Wilson RD., et al. "Pre-conception Folic Acid and Multivitamin Supplementation for the Primary and Secondary Prevention of Neural Tube Defects and Other Folic Acid-Sensitive Congenital Anomalies". Journal of Obstetrics and Gynaecology Canada 37.6 (2015): 534-552.

27. "Fact Sheet for Health Professionals-Folate" (2011).

28. Robitaille J., et al. "National Birth Defects Prevention S. Maternal nutrient intake and risks for transverse and longitudinal limb deficiencies: data from the National Birth Defects Prevention Study, 1997-2003". Birth Defects Research Part A: Clinical and Molecular Teratology 85.9 (2009): 773-779.

29. Smedts HP., et al. "Maternal intake of fat, riboflavin and nicotinamide and the risk of having offspring with congenital heart defects". European Journal of Nutrition 47.7 (2008): 357-365.

\section{Volume 4 Issue 6 June 2021}

\section{(c) All rights are reserved by Mohammed Ayed Huneif and Ramadhan Oruch.}

